\section{Acute transaminitis after initial days of starting haloperidol}

\author{
Rami Gabriel,1 Todd Wojtanowicz, ${ }^{2}$ \\ Reza Farokhpay,1,2 Robert Bota1,2
}

1School of Medicine, and 2Department of Psychiatry, University of California, Irvine, CA, USA

\begin{abstract}
Haloperidol is a first-generation antipsychotic butyrophenone that is lipophilic, readily absorbed, and extensively metabolized in the liver. The occurrence of elevated liver enzymes with haloperidol is reported to be $2.4 \%$ with cases generally occurring in the setting of chronic use. In this case, we present a patient who developed elevated liver enzymes 1-2 days after starting haloperidol treatment on two separate occasions and in the context of negative hepatic viral and autoimmune serology. Liver enzymes consistently had alanine transaminase > aspartate transaminase and peaked at 288 $\mathrm{U} / \mathrm{L}$ prior to discontinuation of the medication. The patient was taken off haloperidol after serology resulted and clozapine regimen started. He was able to tolerate clozapine well with recovery of his transaminitis and psychiatric stabilization.
\end{abstract}

\section{Introduction}

Haloperidol is a first-generation antipsychotic butyrophenone that is lipophilic and readily absorbed with extensive metabolism occurring in the liver and less than $1 \%$ of original drug excreted in urine. ${ }^{1}$ In the liver, haloperidol is $50-60 \%$ metabolized by glucuronidation and approximately $25 \%$ percent by reduction. The remaining $15-30 \%$ are through oxidation reactions. ${ }^{2}$ CYP3A4 has been considered as the major enzyme involved in haloperidol reduction but CYP2D6 has also shown influence in metabolism.3.4 Liver toxicity from haloperidol has been well studied in the chronic setting but few reports show acute effects on the liver. ${ }^{5}$ In this paper, we discuss an episode of transaminitis concordant with starting and restarting haloperidol in the context of negative serology of liver pathology.

\section{Case Report}

A 44-year old man of Southeast Asian origin with a past medical history of substance use disorder (IV methamphetamine and heroin) and schizophrenia presented to the emergency department after being found increasingly disoriented while at a clinic visit. Prior treatment of schizophrenia consisted of paliperidone palmitate $234 \mathrm{mg}$ every 28 days, his last dose was one week from his presentation. While at the psychiatric center at the University of California, Irvine (UCI) the patient continued to have auditory hallucinations $(\mathrm{AH})$, visual hallucinations (VH) and disorganized thinking. Hallucinations included individuals attempting to bite him if he did not put toothpaste across his forehead. The patient was initially started on thiamine and risperidone but after no improvement in symptoms a regimen of haloperidol was started to supplement his long acting injectable. A dose of $10 \mathrm{mg}$ QHS was initiated on $8 / 31$ and then continued until $9 / 3$ when the dose was increased to $15 \mathrm{mg}$ QHS from 9/4 to 9/9. While on treatment, a CMP revealed that the patient had developed a transaminitis that showed an elevated Alkaline Phosphatase, and an Alanine Transaminase (ALT) greater than Aspartate Transaminase (AST). The ALT was three times normal at $166 \mathrm{U} / \mathrm{L}$ on $9 / 7$ from his baseline of 51 taken at 8/27 (Figure 1). A thorough history and physical revealed that the patient had no symptoms of abdominal pain, no icterus, jaundice, hepatomegaly on percussion, or abdominal tenderness. As a precaution, the patient was discontinued from haloperidol on 9/9 and showed improvement in ALT levels down to $100 \mathrm{U} / \mathrm{L}$ two days after (Figure 1). The patient was started on a clozapine taper of $25 \mathrm{mg}$ daily increments with the first $25 \mathrm{mg}$ dose given on $9 / 10$. Despite having improvement in liver function testing, it was still uncertain if the transaminitis was drug-induced or due to underlying liver pathology given patient's history of IV substance abuse. Hepatology was consulted at the time, and a full serologic workup of liver pathology was ordered: Hepatitis A,B, and $\mathrm{C}$; Anti-Smooth Muscle Ab, AntiMitochondrial Ab, Anti-Nuclear Ab. While labs were pending, the patient began to have worsening hallucinations and aggression, so haloperidol was restarted on $9 / 12$ to $9 / 14$ as a $10 \mathrm{mg}$ QHS dose that was tapered to $5 \mathrm{mg}$ QHS on 9/15 and 9/16 (Figure 2). Once again, the AST and ALT levels rose with ALT being higher, reaching a peak of 288 U/L on $9 / 18$. The patient was monitored for several days after stopping haloperidol on 9/16 with a continued clozapine regimen of 200mg QHS. On 9/27 the patient continued to recover from his transaminitis and had an
Correspondence: Robert Bota, University of California, Irvine, UCIMC, Bldg 3, Rt 88, Orange, CA 92868, USA.

Tel.: +1.714.4562056.

E-mail: rbota@uci.edu

Key words: Haldol, Transaminitis, Schizophrenia, Liver toxicity.

Acknowledgements: gratitude is expressed to the UC Irvine Psychiatry Department for staff support and continuing excellence in improving learning and patient care.

Contributions: RG: Drafting manuscript, data collection, data analysis; TW: Patient care, drafting manuscript, data analysis; RF: Overseeing care and project details; RB: Editing manuscript.

Conflict of interest: the authors declare no potential conflict of interest.

Funding: this research was supported with departmental grants from the UC Irvine Psychiatry Department. The sponsors had no role in the design, conduct, or decision to publish the research, nor did they have any role in the review or approval of the manuscript.

Received for publication: 18 March 2019 Accepted for publication: 18 March 2019

This work is licensed under a Creative Commons Attribution-NonCommercial 4.0 International License (CC BY-NC 4.0).

CCopyright R. Gabriel et al., 2019

Licensee PAGEPress, Italy

Mental Illness 2019; 11:8113

doi:10.4081/mi.2019.8113

ALT level of 71. The patient was stabilized on clozapine for psychiatric management and tolerated the new regimen well.

\section{Discussion}

In the patient described above, a notable transaminitis was found on routine labs that was temporally correlated with starting haloperidol while in the psychiatric ward. The patient was tested for serologies for Hepatitis A, B, and C, negative autoimmune hepatitis including Anti-Mitochondrial Ab, Anti-Nuclear Ab, Anti-Smooth Muscle Ab. After stopping the medication and beginning clozapine therapy, the transaminitis correspondingly began to resolve consistent with the hypothesis of an acute drug toxicity secondary to starting haloperidol. Due to extenuating circumstance of the patient's worsening psychosis, recovering transaminitis, and 
pending hepatologic serologies, haloperidol was restarted while clozapine was gradually increased. Subsequently, the patient once again developed a transaminitis and showed recovery after immediately stopping the medication, thereby strengthening evidence for haloperidol toxicity. It is interesting to note that the patient developed a transaminitis more severe when given the second trial. This secondary increased elevation in Liver Function Test (LFT) levels also may suggest a sensitivity reaction with worsening effects with each treatment dose (Figure 3). A report has also shown evidence for hypersensitivity reactions with elevated eosinophils. 6 This patient did not have any significant or abnormal elevation in eosinophils.

The incidence of haloperidol induced hepatitis is studied to be approximately $2.4 \% 7$, however the incidence of acute transaminitis immediately following drug therapy has not been well studied. This is not the first case of an acute haloperidolinduced transaminitis, Ouanes et al. had a similar report showing an eight-fold increase in transaminases (with ALT $>$ AST) 10 days after initiating treatment with haloperidol. 8 This case study adds further support with of an acute haloperidol transaminitis with worsening hepatitis with subsequent trials. The temporal association between initiating haloperidol treatment and the transaminitis, in the context of negative serologies and a controlled environment, yields strong evidence for the consideration of haloperidol as a culpable agent in elevated liver enzymes.

\section{Conclusions}

Acute haloperidol-induced transaminitis is not well studied, with only one case report presuming the drug was the culprit due to the context of negative serology and liver scan. ${ }^{8}$ In this case, we provided temporal relation to starting and stopping haloperidol with the rise and fall of liver enzymes. The patient was asymptomatic throughout the time course, had negative hepatic serologies, and was able to fully recover. The patient was closely monitored for transaminitis despite the scant evidence of an acute haloperidol transaminitis and thus was taken off the medication as much as possible without being detrimental to his psychiatric wellbeing. He tolerated the change to a clozapine well and stabilized psychiatrically and medically on the new regimen.

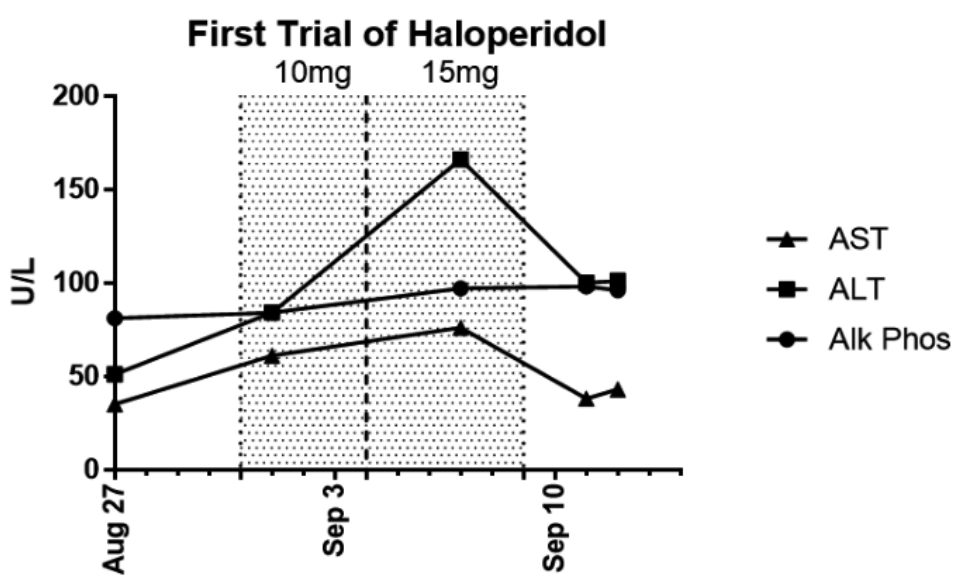

Figure 1. Graphical representation of ALT, AST, and Alkaline Phosphatase (Alk Phos) before and after initially starting haloperidol treatment. The shaded area shows days where patient was taking either $10 \mathrm{mg}$ haloperidol or $15 \mathrm{mg}$ haloperidol.

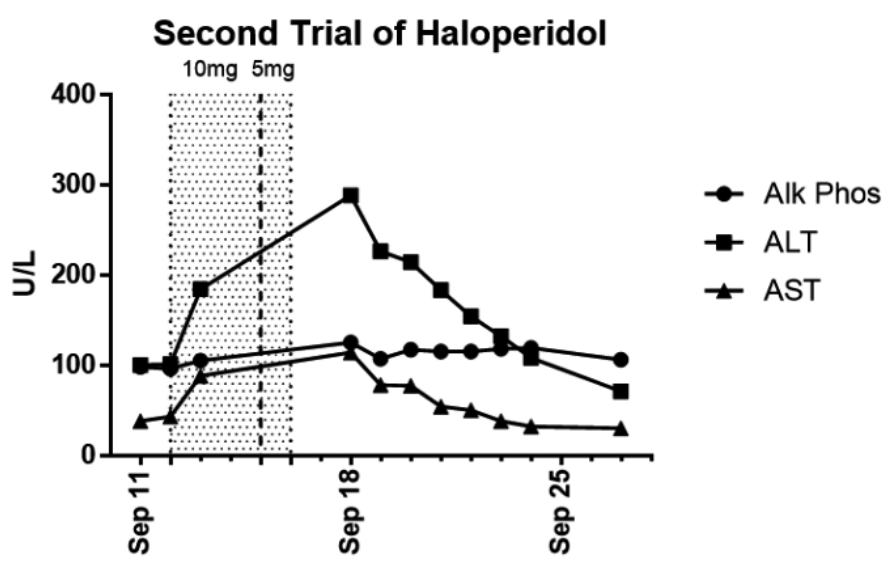

Figure 2. Graphical representation of ALT, AST, and Alkaline Phosphatase before and after second attempt of haloperidol treatment. The shaded area shows days of haloperidol treatment of $10 \mathrm{mg}$ or $5 \mathrm{mg}$.

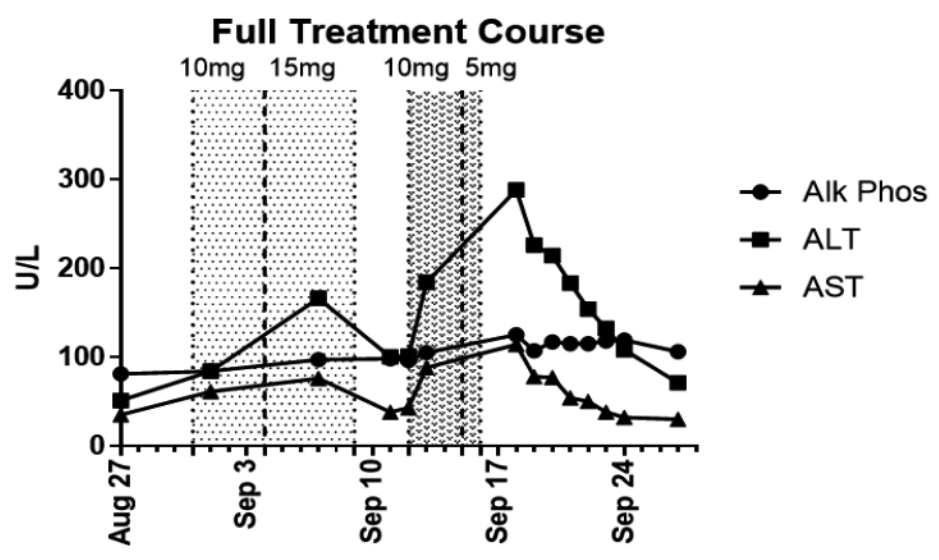

Figure 3. Graphical representation of ALT, AST, and Alkaline Phosphatase throughout treatment course. The shaded area shows days of haloperidol treatment of $10 \mathrm{mg}, 15 \mathrm{mg}$, $10 \mathrm{mg}$, and $5 \mathrm{mg}$ respectively from left to right. 


\section{References}

1. Kudo S, Ishizaki T. Pharmacokinetics of Haloperidol. Clin Pharmacokinet 1999;37:435-56.

2. Greenbaum L, Strous RD, Kanyas K, et al. Association of the RGS2 gene with extrapyramidal symptoms induced by treatment with antipsychotic medication. Pharmacogen Genomics 2007;17:519-28.

3. Titier K, Girodet PO, Verdoux H, et al. Atypical antipsychotics: from potassium channels to torsade de pointes and sudden death. Drug Saf 2005;28:35-51.

4. Kato $\mathrm{Y}$, Nakajima $\mathrm{M}$, Oda $\mathrm{S}$, et al. Human UDP-Glucuronosyltransferase Isoforms Involved in Haloperidol Glucuronidation and Quantitative Estimation of Their Contribution. Drug Metabol Disposit 2011;40:240-8.

5. Vandael E, Vandenberk B, Vandenberghe $\mathrm{J}$, et al. Risk management of QTc-prolongation in patients receiving haloperidol: an epidemiological study in a University hospital in Belgium. Int $\mathrm{J}$ Clin
Pharmacy 2016;38:310-20.

6. Fuller CM, Yassinger S, Donlon P, et al. Haloperidol-induced liver disease. West J Med 1977;127:515-8.

7. Gaertner I, Altendorf K, Batra A, Gaertner HJ. Relevance of liver enzyme elevations with four different neuroleptics: a retrospective review of 7,263 treatment courses. J Clin Psychopharmacol 2001;21:215-22.

8. Ouanes S, Damak R, Hajri M, et al. Haloperidol-induced Hepatitis. Europ Cytolytic 2015;30:1597. 